\title{
Advancing the Science of Dissemination and Implementation in Behavioral Medicine: Evidence and Progress
}

\author{
Carina K. Y. Chan ${ }^{1,2} \cdot$ Brian Oldenburg $^{3} \cdot$ Kasisomayajula Viswanath $^{4,5}$
}

Published online: 22 May 2015

(C) International Society of Behavioral Medicine 2015

\begin{abstract}
The enormous time lag between the discovery of new knowledge and its implementation poses a significant challenge to improving public health because of the very slow uptake into policy and practice. The field of dissemination and implementation research in behavioral medicine is receiving increased attention because of the keen interest in accelerating knowledge transfer from relevant research to improve the health and wellbeing of populations in many different settings, contexts, and countries around the world. This is particularly important in high-risk populations, resource-poor and developing regions of the world where the difference in health systems, languages, and cultures very significantly influences the translation of evidence into policy and practice. Moreover, demonstrating the broader societal and economic value of behavioral interventions in settings where they are implemented can further support the sustainability, uptake, and implementation of these findings in other settings and contexts. This special issue presents a series of empirical studies, reviews, and case studies that address dissemination, implementation, and translation issues in both developed and developing countries. Specifically, the learnings from the application of many and varied theories and research methodologies are very
\end{abstract}

Carina K. Y. Chan

carina.chan@acu.edu.au

1 School of Psychology, Australian Catholic University, 1100 Nudgee Road, Banyo, QLD 4014, Australia

2 Monash University, Bandar Sunway, Malaysia

3 The University of Melbourne, Melbourne, Australia

4 Harvard School of Public Health, Boston, MA, USA

5 Dana Farber Cancer Institute, Boston, MA, USA relevant for bridging the current division between research findings and their translation and uptake into policy and practice.

\section{Introduction}

Behaviors are central to explaining health and many healthrelated outcomes. Specifically, behavior is directly and indirectly related to improving health and the prevention, development, diagnosis, and treatment of diseases [1]. Behavioral medicine is an interdisciplinary field that integrates biomedical, psychosocial, and behavioral perspectives to improve our understanding of how to improve health in individuals and populations.

There have been some great recent advances in behavioral medicine research that have contributed towards improving patient care and improving health in populations around the world. These have included social, behavioral, and community interventions targeting lifestyle risk factors as well as improving healthcare delivery for many different diseases and conditions [1-4]. In particular, successful efforts to prevent and control non-communicable diseases in developed countries over the last 40 years have been very impressive. For example, the death rates from heart disease among middleaged and older adults have fallen by $70 \%$ in the past three decades in countries such as Australia, Canada, the United Kingdom, Finland, and the United States due at least in part to changes in some key lifestyle risk factors such as smoking [5]. Well-conducted diabetes prevention trials in developed countries have also successfully reduced the incidence of type 2 diabetes by addressing common lifestyle risk factors among pre-diabetic individuals [6]. Similar prevention trials have also demonstrated the relative risk reduction of type 2 diabetes in developing nations such as China and India [7], yet the 
evidence in resource-constrained societies still remains very limited [8]. While compelling empirical evidence supports the efficacy and effectiveness of some behavioral interventions, this encouraging evidence does not necessarily lead to changes in the relevant policy and mainstream healthcare delivery [9]. Furthermore, the time lag in translation can be very substantial in developing countries where the disease burden is very considerable [10]. This lag in global uptake and implementation demonstrates how much more we really need to understand about how to close the gap between what we know and what we $d o$ in such countries. If this lag can be reduced, this will make a very big difference to improving global health over the coming years.

Dissemination and implementation (D\&I) research seeks to understand how scientific advances can be most appropriately translated and implemented into policy and practice. Dissemination is an active approach that focuses on the distribution of information and intervention materials to a specific public health or clinical practice audience with the goal to spread knowledge and the associated evidence-based interventions to stakeholders [11]. Dissemination research specifically emphasizes information on how, when, by whom, and under what circumstances will research evidence be distributed to stakeholders such as agencies, organizations, and health workers. Implementation research addresses how empirically supported interventions can be transferred to real-world settings. It emphasizes the integration of research findings and evidence-based intervention into healthcare service and policy [11]. Implementation research highlights the understanding of behaviors among stakeholders at various levels, i.e., individual healthcare consumers, families, communities, and organizations. This is particularly important as many behavioral interventions inevitably involve multiple parties for successful implementation in real-world settings. D\&I science involves multi-disciplinary collaboration that may include, but is not limited to, all of the public and population health sciences, communication science, organizational management, business administration, marketing, behavior change, and economics. D\&I research in behavioral medicine specifically aims to understand, identify, and overcome barriers to adoption of behavioral interventions that address health promotion and disease management.

Appropriate use of theories, models, and frameworks is very important for D\&I science. Theories should be broadly applicable and not content-specific to demonstrate the interrelated concepts and constructs to allow prediction of outcomes. Many theoretical frameworks or models have been reported in the D\&I literature, and they vary in construct flexibility, their focus on D\&I activities, and the level of the socioecological framework at which the model operates (see [12, 13] for reviews). For example, diffusion of innovations, persuasive communication, and social marketing [13] are all theories commonly used in such research. The RE-AIM framework [14] is often used for D\&I planning and evaluation. More recently, the Behaviour Change Wheel [15] provides a comprehensive framework for designing and implementing behavior change interventions by drawing upon a wide range of disciplines and approaches that are relevant to policy makers, practitioners, and researchers. However, there is still a lack of consensus as to which theories - or combinations of theories - are best suited for different settings (primary care, workplace, community) and at various levels (organizational and policy) of change [16].

While randomized controlled trials are highly valued in much health and medical research, other study designs are often more appropriate in D\&I research [17]. Although randomized efficacy and effectiveness trials generally precede D\&I studies on the research to practice continuum [18], public health benefit is not solely determined by the evidence of efficacy or effectiveness for a particular intervention. Instead, the extent to which the interventions can (1) reach a large number of people for the most benefit, (2) be adopted by different settings, (3) be consistently implemented by trained staff personnel, and (4) produce replicable and sustainable effects at reasonable cost are all critically important issues to be addressed. D\&I research focuses more on investigating what strategies can increase the speed of implementation, improve the quality of delivery, and enhance the degree of access or penetration of the interventions [18] rather than whether a strategy or intervention works under ideal conditions.

\section{Barriers in Translating Research into Policy and Practice}

Many factors and influences help explain the poor uptake of evidence into policy and practice. Glasgow and Emmons [19] reviewed some influential factors including the characteristics of the intervention, the situation of the intended target settings, research design, and the interactions of these factors. Firstly, the characteristics of the interventions may not be appealing because many efficacious health promotion interventions are often expensive, labor-intensive, and time-consuming to implement in real-world settings. Secondly, there are also many barriers and challenges in the settings (e.g., worksites, clinics, schools) in which the interventions are to be implemented or scaled up. Challenges such as competing demands, limited resources, time and organizational support, financial and organization (in)stability can influence the decision to adopt evidence-based interventions or even if they are adopted, the quality of delivery may be compromised. Lastly, the characteristics of research designs may also impede successful translation. The use of unrepresentative samples of patients, clinicians and settings, failure to provide process evaluation data, and failure to assess and evaluate implementation and 
maintenance can contribute to loss in translation. Moreover, there is an urgent need to address the context, highlighting the importance of external validity when we design, implement, and evaluate behavior interventions [19]. This is particularly relevant as the field of D\&I science grows; there is more recognition of the tensions between internal and external validity.

To the target audience, the cost of deploying complex processes and strategies, even if they have been proven effective, may still discourage uptake and implementation. Stakeholders or people in the industry are probably more interested in economic gains. Therefore, an economic analysis of D\&I processes and strategies can provide information on the outcomes that a strategy may achieve and its associated cost, which can then be compared against other competing strategies. The idea is to understand the potential attractiveness of a proposed process by quantifying the cost spent on a healthcare good or service relative to outcomes in monetary terms. This is also an area that is rather thin in the D\&I literature in behavioral medicine.

There are still many other areas where D\&I research needs to be undertaken. These include identifying factors that are critical for successful replication of basic research and translation to real-world healthcare settings [20], engaging stakeholders throughout the discovery to practice process [21], funding high-quality investigations that engage users or stakeholders of research [22], making better use of models or frameworks [12], and focusing on external validity and transparency of reporting findings [17].

Since the last decade, the scientific community has given more attention to the process of translating research evidence to public health and clinical practice. Funding bodies such as the U.S. National Institute of Health (NIH) and the U.S. Centers of Disease Control and Prevention (CDC) have begun to provide funding to investigations that can answer questions relevant to end-users of research. Other more global funding opportunities have also become available for implementation research. For example, the Global Alliance for Chronic Disease (GACD) is a network organization of the world's largest public health research funding agencies that support research activities for preventing and controlling chronic diseases, with particular focus on multi-disciplinary programs that address issues in low- and middle-income countries and also vulnerable populations. Involvement with communities and stakeholders often relies on funding availability and continuity. These funding efforts also recognize the need to invest in early development of partnerships with communities especially in settings where resources may be limited and health disparities are large.

The launch of peer-reviewed journals in recent years - e.g., Implementation Science and Translational Behavioral Medicine - that focus on multi-disciplinary and D\&I issues has also demonstrated the increasing priority of D\&I research. The International Society of Behavioral Medicine (ISBM) has also recognized the priority of D\&I research and facilitated its first satellite forum on dissemination and implementation at its biennial International Congress of Behavioral Medicine (ICBM) in 2012 in Hungary and subsequently in 2014 in The Netherlands. Two major outcomes of these satellite meetings have been the launch of the current special issue and the formation of a global network on D\&I research.

\section{Current Special Issue}

The 15 articles in this special issue address many of the issues that have already been discussed in this editorial and which are associated with the challenges and time lag in translating research discoveries into policies and practice to improve public health. They present some of the available evidence on how dissemination, implementation, and translation may be achieved in clinical, organizational, and community settings across different developed and developing regions. Some common barriers and factors important for consideration when conducting D\&I research are also highlighted.

To accelerate research evidence to end-users, settings, and communities, information needs to be transmitted to the targeted audience and settings. One area of D\&I research is to examine how best the information can be transferred to different parts of the society to be usable. Systems thinking is an approach that can be used to study complex health issues, and network analysis is a system science tool that can help understand the structural and relational properties of the travel of information. Burke, Lich, Neal, Meissner, Yonas, and Mabry [23] demonstrate how systems science methods can be used to address D\&I challenges associated with chronic disease prevention, community violence prevention, and education intervention. Gainforth, Latimer-Cheung, Moore, Athanasopoulos, and Martin Ginis [24] show how network analysis could be applied to transferring knowledge to community-based organizations. In their study, they use network analysis to disseminate physical activity guidelines to people with spinal cord injury.

Very often, health behavior change may be modified through the implementation of policy and environmental change interventions (e.g., smoke free policy, food calories labeling). To facilitate this implementation process, evidence-based interventions need to be disseminated in order to reach out to the intended audiences. Using case examples to address physical activity, healthy eating, and tobacco product sales, Leeman, Myers, Ribisl, and Ammerman [25] identify some key strategies (e.g., identifying the best available evidence, disseminating intervention menus, building capacity) for promoting access and usage by practitioners. 
Community settings have the potential to play an important role in the widespread application of evidence-based interventions and policies. Stoutenburg, Stanzilis, and Falcon [26] review physical activity and dietary modification programs in community settings and have observed varying levels of success. The authors describe common features and barriers that the translation process faced. One suggestion made was to better identify barriers to program implementation such as challenges to recruit and work with local communities.

Indeed, involvement and participation of community and other stakeholders is essential for improving the quality and relevance of research. One method to encourage this is by using participatory approach in D\&I research. Pinto, Waldemore, and Rosen [27] have undertaken a randomized controlled trial with a community-based organization to promote exercise among cancer survivors using peer support in New England, USA. They report the process and challenges of this collaboration, and identify some important issues that should be considered and addressed to facilitate future engagement of community partners. Also, using a participatory approach, Tomasone, Martin Ginis, Esterbooks, and Domenicucci [28] report the restructuring of a curriculum to encourage health professionals to prescribe physical activity to those with physical disabilities in Canada. The collaborative development of the curriculum and subsequent assessment of the interventionists' cognitions for adoption showed promising future uptake and implementation.

One important aspect of D\&I research is to assess whether the evidence-based interventions being adopted by individuals and organizations are indeed the ones that are known to be effective. If they are not, then the implemented interventions may not be likely to benefit the target population(s). To achieve the optimal benefits, it is critical that the efficacious interventions are implemented with sufficient fidelity and consistency. Augustsson, von Thiele Schwarz, Stefors-Hayes, and Hasson [29] have investigated the differences in implementation fidelity of an occupational health intervention in an organizational setting in Sweden. They report variations in fidelity within different organizational units and suggest that study design and implementation strategies may need to be adapted to suit local context.

Behavioral interventions that can reach out to the target populations and minimize attrition have higher chances of benefiting those who need them. Some key indicators during the implementation process are extremely critical for scaling up to wider populations. Sainsbury, Mullan, and Sharpe [30] have conducted an online intervention for people with coeliac disease with the aim to improve gluten-free diet adherence. Their feasibility trial sheds light on how changes to the structure and implementation of their intervention may minimize attrition and enhance effectiveness for future scale-up of similar health promotion effort. Also, using a web-based delivery, Ramadas, Chan, Oldenburg, Hussain, and Quek [31] have culturally adapted and undertaken a trial of a dietary modification program for type 2 diabetes management in Malaysia. The content of the dietary program emphasizes the cultural appropriateness of food among the major ethnic groups in the country. Adaptation of empirically supported interventions in different ethnic and cultural groups in ways that aim to reflect the corresponding cultural norms, values, and beliefs of particular groups can enhance their acceptability and relevance to the targeted populations.

One important area for dissemination and implementation research is to establish external validity of efficacious health interventions and to identify strategies to adapt and replicate successful trials in high-risk populations and low-resource systems where health disparities exist [16]. This is particular relevant in the context of preventing and controlling for chronic diseases. For example, while other empirical studies have demonstrated the effectiveness of peer support in facilitating some health behavior changes and promoting better diabetes management [3], most studies were limited to developed countries and structured within more advanced health systems.

Baumann, Frederick, Betty, Jospehine, and Agatha [32] report the feasibility of a nurse-led peer support intervention to manage type 2 diabetes in rural Uganda, emphasizing some setting-specific conditions that were critical for future intervention uptake. Using the train-the-trainers model, Jones, Weiss, and Chitalu [33] evaluated the challenges for implementing an efficacious HIV prevention program in Zambia, Sub-Saharan Africa. The multi-level, context-specific issues pertaining to implementation are identified, with an emphasis of the importance of the combination of some key factors such as collaborative structure, leadership, commitment, and support at the government level that are critical for its adoption. In an attempt to address the very big challenge of cardiovascular diseases in China, Zhong, Potemans, Zhang, and Oldenburg [34] utilized a screening tool adapted from the Netherlands to identify residents at risk and implemented a prevention program in seven communities in Anhui province. The use of local community health service centers and participants was found to be a very feasible way of delivering a cardiovascular disease prevention program in that region of China.

All of these reported studies highlight the complexity of the translation process of previously tested screening tools and behavioral interventions and that this process requires considerable contextualization and adaptation to the local environment and culture in order to be implementable, sustainable, and scalable in these new contexts. A number of the studies demonstrate that the involvement of many different groups of stakeholders-including health professionals, local health workers in communities, and community participants - is also critically important for successful implementation in developing countries.

Implementation research in healthcare settings including health service delivery, changing health professionals 
behaviors, and clinical decision making, has great potential to stimulate policy and practice interest, yet the quality and implementation gap remains when we look at performance and impacts of healthcare delivery and services. Focusing on the process and the contextual factors relevant for effective strategy may be more important than simply addressing if certain strategies are effective. Huijg, van der Zouwe, Crone, Verheijden, Middelkoop, and Gebhardt [35] report a qualitative study exploring the stakeholders' perceptions on the factors that would affect the introduction of physical activity interventions in the primary healthcare setting. Findings from the interviews highlight the important factors from stakeholder perspectives to be considered before the development of an intervention.

Weiss, Tobin, Lopex, Simon, Cook, and Jones [36] report a successfully translated behavioral intervention (SMART/EST Women's Program) which aimed at reducing distress, improving health, and decreasing risk behaviors for disadvantaged women living with HIV/AIDS in the USA. Using the REAIM framework, their study has shown the processes involved in translating evidence to clinical practice by using Community Health Center staff (verus research staff) to conduct the intervention. More encouragingly, not only did the study demonstrate equivalence of outcomes between the research-led and community health center staff-led groups, for some, the results favored the community staff-led group.

Lastly, while it is important to understand and identify strategies and factors that can improve dissemination and implementation, very limited research has taken an economic evaluation approach in D\&I research, especially in the context of behavioral interventions. The economic perspective of utilizing a behavioral intervention can provide important information with regards to its potential sustainability and identify the return on investment to organizations or groups who will adopt and maintain the program. Brown, Cameron, and Ramondt [37] used a hypothetical scenario on breast cancer rehabilitation program for women to present how budget impact analysis can assess the change in monetary values relative to outcomes for the organization and participants. Some key economic considerations for future sustainable behavioral interventions were also recommended by the authors.

\section{Summary}

Optimizing dissemination, adoption, and delivery of successful intervention in clinical, community, and public health settings are central to dissemination and implementation science [38]. Narrowing the gap between what we know and do in order to improve and maximize the health and wellbeing of individuals and populations is the ultimate goal of this burgeoning new field. The 15 papers in this Special Issue provide some excellent examples of how the science of dissemination and implementation can be applied in the global field of behavioral medicine. The work presented covers diverse populations, settings, and methodologies, with the majority of them strongly informed by theoretical frameworks. There are many findings from these studies that are also relevant for further adaptation and refinement to inform future policy and practice. This special issue notwithstanding, the gap between research, policy, and practice is still far from acceptable, so much more research is required in this field, particularly as applied to the very big public health challenges that face low- and middle-income countries. In particular, more collaborative research undertaken in partnership with practitioners, policymakers, and citizens will provide more learning that are amenable and feasible for wider implementation and uptake into policy and practice.

Conflict of Interest Carina K. Y. Chan, Brian Oldenburg, and Kasisomayajula Viswanath declare that there is no conflict of interest.

\section{References}

1. Fisher EB, Fitzgibbon ML, Glasgow RE, Haire-Joshu D, Hayman LL, Kaplan RM, et al. Behavior matters. Am J Prev Med. 2011;40 (5):e15-30.

2. Oldenburg B, Absetz P, Chan C. Behavioral interventions for prevention and management of chronic disease. In: Steptoe A, Freedland K, Jenning JR, Lllabre M, Manuck S, Susman E, editors. Handbook of behavioral medicine: methods and applications. New York: Springer; 2010. p. 969-88.

3. Fisher EB, Boothroyd RI, Coufal MM, Baumann LC, Mbanya JC, Rotheram-Borus MJ, et al. Peer support for self-management of diabetes improved outcomes in international settings. Health Aff. 2012;31(1):130-9.

4. Absetz P, Oldenburg B, Hankonen N, Valve R, Heinonen H, Nissinen A, et al. Type 2 diabetes prevention in the real world: three-year results of the GOAL lifestyle implementation trial. Diabetes Care. 2009;32(8):1418-20.

5. World Health Organization. Preventing chronic diseases: a vital investment. WHO global report [Internet]. 2005. Available from: http://www.who.int/chp/chronic_disease_report/en/.

6. Hussain A, Claussen B, Ramachandran A, Williams R. Prevention of type 2 diabetes: a review. Diabetes Res Clin Pract. 2007;76(3):317-26.

7. Ramachandran A, Snehalatha C, Mary S, Mukesh B, Bhaskar AD, Vijay V. The Indian Diabetes Prevention Programme shows that lifestyle modification and metformin prevent type 2 diabetes in Asian Indian subjects with impaired glucose tolerance (IDPP-1). Diabetologia. 2006;49(2):289-97.

8. Rawal LB, Tapp RJ, Williams ED, Chan C, Yasin S, Oldenburg B. Prevention of type 2 diabetes and its complications in developing countries: a review. Int J Behav Med. 2012;19:121-33.

9. Oldenburg B, Absetz P. Lost in translation: overcoming the barriers to global implementation and exchange of behavioral medicine evidence. Transl Behav Med. 2011;1(2):252-5.

10. Beaglehole R, Horton R. Chronic diseases: global action must match global evidence. Lancet. 2010; 376(9753):1619-21.

11. U. S. National Institutes of Health (OBSSR). Dissemination and implementation 2015 [cited 201531 January 2015]. Available 
from: http://obssr.od.nih.gov/scientific_areas/translation/ dissemination and implementation/.

12. Tabak RG, Khoong EC, Chambers DA, Brownson RC. Bridging research and practice: models for dissemination and implementation research. Am J Prev Med. 2012;43(3):337-50.

13. Wilson P, Petticrew M, Calnan M, Nazareth I. Disseminating research findings: what should researchers do? A systematic scoping review of conceptual frameworks. Implement Sci. 2010;5(1):91.

14. Glasgow RE, McKay HG, Piette JD, Reynolds KD. The RE-AIM framework for evaluating interventions: what can it tell us about approaches to chronic illness management? Patient Educ Couns. 2001;44(2):119-27.

15. Michie S, van Stralen M, West R. The behaviour change wheel: a new method for characterising and designing behaviour change interventions. Implement Sci. 2011;6(1):42.

16. Brownson RC, Dresinger M, Colditz GA, Proctor EK. The path forward in dissemination and implementation research. 2012. In: Dissemination and implementation research in health: translating science to practice [Internet]. Oxford University Press. Available from: doi:10.1093/acprof:oso/9780199751877.003.0024.

17. Glasgow RE. What types of evidence are most needed to advance behavioral medicine? Ann Behav Med. 2008;35(1):19-25.

18. Landsverk J, Brown CH, Chamberlain P, Ogihara M, Czaja S, Goldhaber-Fiebert JD, et al. Design and analysis in dissemination and implementation research. 2012 [cited 4 February, 2015]. In: Dissemination and implementation research in health: translating science to practice [Internet]. Oxford University Press, [cited 4 February, 2015]. Available from: doi:10.1093/acprof:oso/ 9780199751877.003.0012.

19. Glasgow RE, Emmons KM. How can we increase translation of research into practice? Types of evidence needed. Annu Rev Public Health. 2007;28:413-33.

20. Chalmers I, Bracken MB, Djulbegovic B, Garattini S, Grant J, Gülmezoglu AM, et al. How to increase value and reduce waste when research priorities are set. Lancet. 2014;383(9912):156-65.

21. Lobb R, Colditz GA. Implementation science and its application to population health. Annu Rev Public Health. 2013;34:235-51.

22. Ioannidis JPA, Greenland S, Hlatky MA, Khoury MJ, Macleod MR, Moher D, et al. Increasing value and reducing waste in research design, conduct, and analysis. Lancet. 2014;383(9912):166-75.

23. Burke JG, Lich K, Neal J, Meissner H, Yonas M, Mabry P. Enhancing dissemination and implementation research using systems science methods. Int J Behav Med. 2014;1-9.

24. Gainforth HL, Latimer-Cheung AE, Moore S, Athanasopoulos P, Martin Ginis KA. Using network analysis to understand knowledge mobilization in a community-based organization. Int J Behav Med. 2014;1-9.

25. Leeman J, Myers A, Ribisl K, Ammerman A. Disseminating policy and environmental change interventions: insights from obesity prevention and tobacco control. Int J Behav Med. 2014;1-11.
26. Stoutenberg M, Stanzilis K, Falcon A. Translation of lifestyle modification programs focused on physical activity and dietary habits delivered in community settings. Int J Behav Med. 2014;1-16.

27. Pinto BM, Waldemore M, Rosen R. A community-based partnership to promote exercise among cancer survivors: lessons learned. Int J Behav Med. 2014;1-8.

28. Tomasone JR, Martin Ginis KA, Estabrooks PA, Domenicucci L. Changing minds, changing lives from the top down: an investigation of the dissemination and adoption of a Canada-wide educational intervention to enhance health care professionals' intentions to prescribe physical activity. Int J Behav Med. 2014;1-9.

29. Augustsson H, von Thiele Schwarz U, Stenfors-Hayes T, Hasson $\mathrm{H}$. Investigating variations in implementation fidelity of an organizational-level occupational health intervention. Int J Behav Med. 2014;1-11.

30. Sainsbury K, Mullan B, Sharpe L. Dissemination of an online theory-based intervention to improve gluten-free diet adherence in coeliac disease: the relationship between acceptability, effectiveness, and attrition. Int J Behav Med. 2014;1-9.

31. Ramadas A, Chan CKY, Oldenburg B, Hussien Z, Quek KF. A webbased dietary intervention for people with type 2 diabetes: development, implementation, and evaluation. Int J Behav Med. 2014;1-9.

32. Baumann LC, Frederick N, Betty N, Jospehine E, Agatha N. A demonstration of peer support for Ugandan adults with type 2 diabetes. Int J Behav Med. 2014;1-10.

33. Jones D, Weiss S, Chitalu N. HIV prevention in resource limited settings: a case study of challenges and opportunities for implementation. Int J Behav Med. 2014;1-9.

34. Zhong X, Potemans B, Zhang L, Oldenburg B. Getting a grip on NCDs in China: an evaluation of the implementation of the DutchChina cardiovascular prevention program. Int $\mathrm{J}$ Behav Med. 2014;1-11.

35. Huijg JM, van der Zouwe N, Crone MR, Verheijden MW, Middelkoop BJC, Gebhardt W. Factors influencing the introduction of physical activity interventions in primary health care: a qualitative study. Int J Behav Med. 2014;1-11.

36. Weiss SM, Tobin JN, Lopez M, Simons H, Cook R, Jones DL. Translating an evidence-based behavioral intervention for AIDS into clinical practice: the SMART/EST women's program. Int J Behav Med. 2014;1-10.

37. Brown PM, Cameron LD, Ramondt S. Sustainability of behavioral interventions: beyond cost-effectiveness analysis. Int J Behav Med. 2014;1-9.

38. Brownson RC, Colditz GA, Proctor EK. Dissemination and implementation research in health: translating science to practice. Oxford University Press; 2012. Available from: http://www. oxfordscholarship.com.ezproxy1.acu.edu.au/view/10.1093/acprof: oso/9780199751877.001.0001/acprof-9780199751877. 